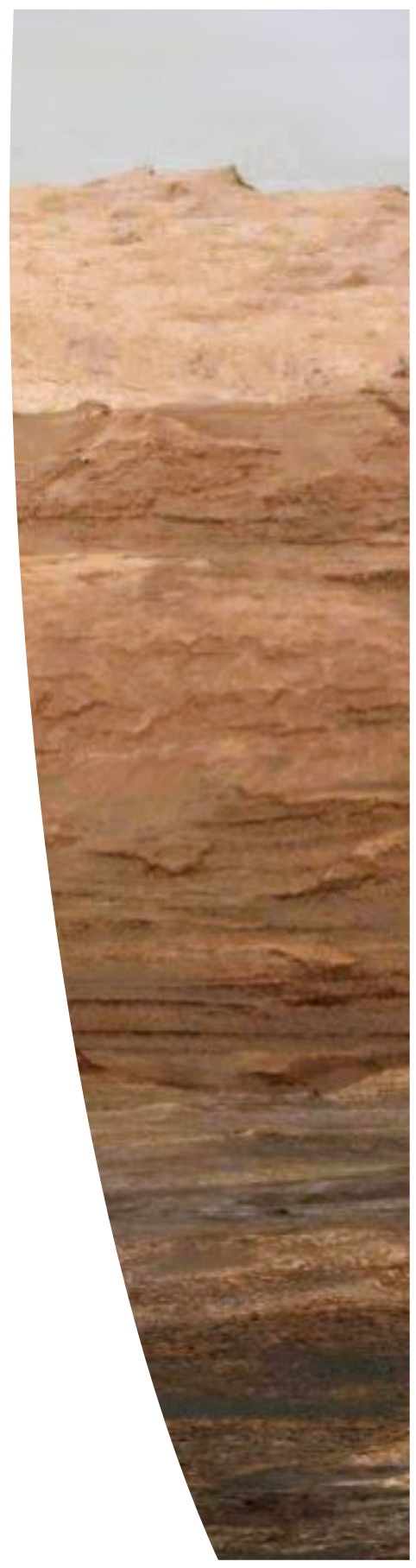

\title{
The evolution of habitable environments on terrestrial planets: Insights and knowledge gaps from studying the geologic record of Mars
}

\author{
A White Paper for the 2023-2032 Planetary \\ Science and Astrobiology Decadal Survey
}

\section{Coordinating Author:}

Briony Horgan, Purdue University

\section{Co-Authors:}

Janice L. Bishop, SETI Institute

Adrian Brown, NASA Headquarters

Wendy Calvin, University of Nevada - Reno

Christopher Edwards, North. Arizona Univ.

Abigail Fraeman, Jet Propulsion Laboratory, California Institute of Technology Tim Goudge, University of Texas at Austin

Linda C. Kah, University of Tennessee

Edwin Kite, University of Chicago

Kennda Lynch, Lunar \& Planetary Institute/USRA

Ramses M. Ramirez, Earth-Life Science Institute

Elizabeth Rampe, Johnson Space Center

William Rapin, Sorbonne Universite

Melissa Rice, Western Washington University

Frances Rivera-Hernández, Dartmouth/Georgia Tech

Kathryn Stack, Jet Propulsion Laboratory, California Institute of Technology Jesse Tarnas, Brown University

Allan Treiman, Lunar \& Planetary Institute/USRA

Christina Viviano, JHU/Applied Physics Laboratory 


\section{Co-Signatories:}

Elena Amador, NASA JPL/Caltech

Andrew Annex, Johns Hopkins University

Ryan Anderson, USGS Astrogeology SC

P. Douglas Archer, Jr., Jacobs/NASA JSC

Ray Arvidson, Washington Univ. St. Louis

Jonathan Bapst, Jet Propulsion Laboratory

Patricio Becerra, University of Bern

Candice Bedford, Lunar \& Planetary Inst./USRA

Jim Bell, Arizona State University

Kristen Bennett, USGS Astrogeology SC

Michael Bramble, Jet Propulsion Laboratory

Edward Cloutis, University of Winnipeg

Matt Chojnacki, University of Arizona

Robert A. Craddock, Smithsonian Institution

Andy Czaja, University of Cincinnati

Lauren Edgar, USGS Astrogeology SC

Alberto G. Fairén, Centro de Astrobiología

Amber L. Gullikson, USGS Astrogeology SC

James Haber, Purdue University

Jennifer Hanley, Lowell Observatory

James W. Head, Brown University

Christopher H. House, Penn. State Univ.

Jeff Johnson, JHU/Applied Physics Laboratory

Devanshu Jha, MVJ College of Engineering

Suniti Karunatillake, Louisiana State University

Corbin Kling, North Carolina State University
Rachel Kronyak, Jet Propulsion Laboratory

Mathieu Lapotre, Stanford University

Kevin Lewis, Johns Hopkins University

Juan M. Lorenzo, Louisiana State University

Nicolas Mangold, CNRS/Université Nantes

Amy McAdam, NASA Goddard SFC

Tom McCollom, Univ. of Colorado, Boulder

Scott Murchie, JHU/Applied Physics Laboratory

Eldar Noe Dobrea, Planetary Science Institute

Marisa Palucis, Dartmouth College

Ashley Palumbo, Brown University

François Poulet, CNRS/Université Paris-Saclay

Deanne Rogers, Stony Brook University

Amanda Rudolph, Purdue University

Catherine Russell, University of Leicester

Alicia M. Rutledge, Northern Arizona University

Mark Salvatore, Northern Arizona University

Noel Scudder, Purdue University

Rachel Sheppard, Jet Propulsion Laboratory

Prakhar Sinha, Purdue University

Rebecca Smith, Stony Brook University

Evan Stanish, University of Winnipeg

Michael Veto, Ball Aerospace

Roger C. Wiens, Los Alamos National Lab.

Rebecca Williams, Planetary Science Institute James Wray, Georgia Institute of Technology

\section{Acknowledgements:}

Part of this research was carried out at the Jet Propulsion Laboratory, California Institute of Technology, under a contract with the National Aeronautics and Space Administration (80NM0018D0004). This white paper is pre-decisional information, for planning and discussion purposes only. 
Summary: Mars is currently the only planet where the surface is known to have made the transition from habitable to uninhabitable. We have made enormous strides in determining the range of habitable environments that existed on ancient Mars, but there is now a pressing need for new data to understand how these environments evolved over Mars history (their timing, persistence, and distribution), to constrain the conditions under which they formed (e.g., climate, water properties, and diagenesis), and ultimately to determine the driving mechanisms for environmental change. Because these environments are records of broader Mars surface and subsurface conditions, constraining their boundary conditions is critical for testing global models of Mars' environmental evolution, past climate, and surface and subsurface habitability. Current models for Mars disagree on which mechanisms could have warmed the surface enough to permit liquid water, why surface habitability apparently switched on and off repeatedly, why Mars dried out, and how deep the desiccation penetrated into the subsurface. Filling these fundamental knowledge gaps using new data from Mars is essential to provide context for Mars Sample Return and provides our best chance to understand what factors in planetary evolution lead to sustained habitable environments on Earth-like worlds. In addition to ongoing support for MSL, new data from other habitable paleoenvironments across Mars is required to make significant progress, and we recommend that the most critical missions are high-resolution orbital spectrometers and small mobile landed missions to a variety of ancient environments. This approach is similar to MASWG Mission Arc 1, "Diverse Ancient Environments \& Habitability." We endorse the MASWG vision for a rejuvenated Mars Exploration Program in the next decade, based on the compelling assertion that "Mars has a uniquely accessible archive of the long-term evolution of a habitable planet."

\section{Introduction}

The Mars Exploration Program has uncovered evidence for an astounding variety of ancient aqueous environments across Mars. High-resolution orbital imaging and spectroscopy have enabled characterization of the meter to decameter-scale geomorphology, mineralogy, and stratigraphy of many of these locations. In addition, landed missions have provided detailed evidence for a complex history of multiple aqueous environments that are not apparent from orbital data. We now know that ancient Mars had aqueous environments perhaps as varied as those on the early Earth, and that these environments on Mars were likely capable of both hosting life and preserving signs of life. However, the bigger picture still remains frustratingly elusive - what factors in planetary evolution lead to sustained habitable environments? Even though we know that habitable environments existed, we lack robust constraints on their timing and duration, the climate/atmospheric conditions under which they existed, how those conditions changed over time, or how their presence and preservation were influenced by other large-scale processes.

Habitability is central to Mars science because developing planet-wide models for the evolution of surface and subsurface conditions governing habitable environments places specific constraints on the geological evolution of the Mars system. Habitability is inherently linked to fundamental planetary processes, including the dynamo, volcanism, impacts, hydroclimate, and the atmosphere. The importance of a continued focus on habitability is reflected in the MEPAG goals document, as habitability and aqueous environments remain one of the central themes for proposed investigations across all of the goals (1). Resolving these questions is also critical for understanding the factors that control habitability of terrestrial planets in our solar system and beyond. Additional data from Mars will enable us to move past the simple model of the Habitable Zone to a more robust understanding of planetary evolution and its influence on the habitability of Earthlike planets over time (2). Thus, interrogating the record of habitable environments on Mars must continue to be an essential goal of the Mars Exploration Program in the decade to come. 
Progress Since Previous Decadal Survey

At the time of the last Decadal Survey, MRO, Mars Odyssey, Mars Express, and MER had revealed clear indicators of past aqueous environments. Spectral detections of diverse aqueous minerals in ancient terrains (3-4) were complemented by high-resolution imaging and topography that revealed landforms and sedimentary deposits associated with surface liquid water on early Mars (5-9). Since then, we have made major advances in our understanding of past environments:

(1) Ancient Mars hosted habitable environments nearly as diverse as those on early Earth. Evidence has accumulated for a wide variety of surface and subsurface environments on Mars. While precipitation-derived surface runoff fed many of the Noachian lakes and rivers identified in orbital imagery (10-12), groundwater was likely the dominant water source for many lakes in the Hesperian (13-15). The precipitation that fed fluviolacustrine systems at the end of the Noachian also produced widespread surface weathering in the form of deep leaching profiles (16-17), some of which show evidence for possible surface wetlands, subsurface aquifers, and climatic transitions (18-21). Subsurface impact-driven hydrothermal systems were detected by Opportunity and mapped widely by CRISM (22-24), and sub-aqueous hydrothermal systems may have been present in Noachian lakes and seas (25). More generally, we now know that subsurface fluids were common on Mars. Alteration by multiple generations of diagenetic fluids is apparent in rover and orbital observations of filled fractures of varying styles and scales (26-29). Fracture-hosted deep subsurface groundwaters on Mars may have been similar to those that exist in Earth's crust and may have provided a stable habitat after the surface of Mars became uninhabitable (30-32).

(2) Sedimentary rocks on Mars record complex histories of deposition, alteration, and erosion. Orbital sub-meter-scale imaging and meter-scale topography have revealed a diverse suite of ancient environments (e.g., aeolian, fluvial, and fans/delta systems; 33-38) that have yielded new insights into the timing/duration, sediment transport, surface conditions, and evolution of martian sedimentary systems (39-43). We also received our first in situ look at a thick sedimentary sequence on Mars from MSL in Gale crater. The Curiosity rover has climbed through hundreds of meters of sediments deposited in habitable fluviolacustrine environments that potentially persisted for $>10^{6}$ years (44-45). Clay and sulfate minerals in the lacustrine sedimentary bedrock are primarily attributed to in situ weathering and authigenic precipitation (46-50), and, with a lack of evidence for ice, suggest relatively warm climates during deposition (51-52). Lake activity may have persisted long after deposition of the strata investigated to date by MSL, based on detection of alteration minerals at higher elevations (53-55) and geomorphic evidence for lakes post-dating erosion of Mt. Sharp (56). Lacustrine sediments also exhibit evidence for multiple phases of diagenesis by groundwaters of varying chemistry (26-27). One mudstone gave a jarosite K-Ar date of $2.12 \pm 0.36 \mathrm{Ga}(57)$, suggesting groundwater was present, at least intermittently, for more than $1 / 2$ of Mars' history. Thus, Gale crater's sedimentary record preserves a complex history of interactions between deposition, alteration, and erosion extending across billions of years. Disentangling how habitability varied over time requires a detailed understanding of this complex history.

(3) Alteration minerals provide key constraints on past environmental conditions on Mars. Many key geochemical indicator minerals have been detected on Mars in the past ten years (5859). For example, phases like akaganeite and jarosite that indicate strongly oxidizing environments over a range of $\mathrm{pH}$ and salinity have been detected both from orbit and in situ observations (6061). Phases inferred to reflect reducing conditions or strong redox gradients include reduced and oxidized sulfur and carbon species detected by MSL (63-60); mixed valence Fe-sulfates and Fephyllosilicates in Mawrth Vallis $(18,19)$; and serpentine $(67)$. These minerals suggest chemical gradients that could have enabled diverse microbial metabolic pathways. 
(4) The ancient atmosphere was likely much thicker than today. A thicker ancient atmosphere has long been postulated for Mars, but this has only recently been confirmed through measurements of isotope ratios by MSL and escape rates by MAVEN. Both indicate that the atmospheric pressure was once much higher, with as much as 1.5-3 bars of $\mathrm{CO}_{2}$ removed from the atmosphere since the Noachian (68) and loss of a global equivalent water layer on the order of 50-100 meters since the Hesperian (69). These observations support the model of a thick $\mathrm{CO}_{2}$ atmosphere and abundant water on early Mars that could have enabled persistent habitable surface environments.

\section{Current Challenges}

We have made major breakthroughs in our understanding of what habitable environments were possible on Mars; however, we have not yet been able to determine the how, when, or why of environmental evolution across Mars due to several major challenges listed below. This lack of a coherent framework for the record of habitable environments means that we also have not been able to constrain which factors in planetary evolution lead to sustained habitable environments.

(1) The complex history of aqueous processes on Mars can make untangling the sequence of events at any given location challenging. The sedimentary record of Mars is the integrated result of many episodes of deposition and erosion, and chemical records of early environments are frequently overprinted by diagenetic processes. Untangling these events on Earth usually requires both detailed outcrop context and microscale analysis (70), both of which are not possible with current orbital data, and the latter is challenging or not possible with current landed data.

(2) Locations where we have a detailed understanding of environments are very localized, without clear constraints on timing or links to planetary evolution. Placing landed observations in a broader stratigraphic framework is challenging because dust cover prevents orbital mapping of relevant minerals and leads to difficulties in understanding the geologic relationships between non-contiguous outcrops in orbital images. Furthermore, without detailed geochronology, placing paleoenvironments into an absolute timeline is challenging. For example, while the Columbia Hills preserve indications of a variety of habitable environments (71-73), their relative and absolute timing are both poorly constrained, and because of dust and lava flow cover, their regional context remains ambiguous. Thus, it is unclear how to place Spirit results in a broader history of Mars.

(3) The environments preserved in extensive altered units are still poorly understood. We have not yet visited regionally/globally extensive altered units with landed missions and their origins remain ambiguous. Efforts to correlate "reference sections" of sedimentary rocks and minerals across Mars using orbital data have had limited success (74). For example, extensive Fe/Mgsmectites have been attributed to groundwater, steam alteration, hydrothermalism, marine alteration, weathering, acid alteration, and impacts $(16,24,75-79)$. Carbonate may have formed in lacustrine, hydrothermal, or arid environments (80-86). Although these units could provide important tie points in planetary evolution, their nature, timing, and significance are poorly constrained.

(4) Paleoclimate models are still too poorly constrained to provide clear context for the geologic record. Paleoclimate models have the potential to provide a framework for interpreting the history of habitability, but we have yet to adequately test model predictions using geologic observations. The mineralogy and geomorphology of Noachian terrains suggest at least $10^{2}-10^{6}$ years of aqueous activity $(16,21,24,41,87-89)$ that continued episodically into the Hesperian and Amazonian (41). However, Noachian paleoclimate models range from persistently cold with rare liquid water $(39,90-94)$ to warm periods $<10^{7}$ years $(87-88)$, depending on poorly constrained factors such as atmospheric composition and water inventory. Additional data is needed to determine how geologic data and paleoclimate models correlate spatially and temporally, and whether or not our assumptions regarding surface conditions and properties of the early atmosphere are correct. 
Knowledge Gaps

These challenges have made it difficult to develop a framework for the nature of environmental transitions on Mars. Knowledge gaps regarding ancient environments fall into several key areas:

Timeline. (1) When was liquid water abundant on the martian surface? Both the relative and absolute timing of surface environments are poorly constrained, which is critical for determining when Mars was habitable and what processes affected habitability. Valley network activity peaked in the late Noachian, but sporadic wet periods may have extended late in Mars history (41). For example, lake activity in Gale crater likely occurred in the Hesperian (95-96), but alluvial activity may have continued into the Amazonian (97). Sulfate and silica deposits are also found in Late Hesperian to Amazonian-aged terrains (98). Late wet events challenge our understanding of the Habitable Zone, because habitability near the outer edge of the habitable zone is assumed to require a thick atmosphere, but Mars in the Amazonian probably had a $\mathrm{CO}_{2}$ partial pressure $<<1$ bar.

(2) What was the duration of habitable surface environments? It is unclear how long and how often wet environments existed through martian history, which has implications for both the establishment of life and its adaptation and evolution under changing surface conditions. Gale crater lake activity could have persisted for up to $10^{6}$ years based on comparisons to Earth-based sedimentation rates (45,99-101), but these estimates are difficult to assess from orbital data. Estimates for construction of the Eberswalde delta range from 10-10 $0^{6}$ years $(12,102)$, and for the Jezero delta, $10-10^{5}$ years (103), depending on widely variable assumptions about intermittency of the lakes. Our knowledge of the duration of habitable surface environments is thus limited to the locations explored by landed spacecraft, and even this is limited by our lack of quantitative geochronology.

(3) How did timing differ for subsurface environments? Subsurface environments are critical for understanding the habitability of Mars over time, as they could have provided a long-term refuge for life. Some timing inferences can be made when diagenetic alteration is observed within outcrops or crater ejecta with constrained ages. However, this is only an upper limit, as pre-impact alteration vs. impact hydrothermalism are difficult to distinguish remotely (104). K-Ar dating of alteration phases in Gale suggests subsurface aqueous environments existed for $>1$ Gyr (57).

Distribution. (1) What is the spatial (lateral and vertical) distribution of habitable environments? We have identified physical and chemical signatures of diverse aqueous environments on Mars (58). However, due to the limited spatial resolution of current data sets, it is not well constrained how common these environments were and what their spatial distribution may have been. This is important for characterizing planetary trends in habitability and the interconnectedness of past environments, which may have impacted the persistence and resilience of ecosystems. It is currently unclear which mineral records correspond to surface, shallow subsurface, or deep subsurface environments. Shallow vs. deep subsurface is important in relating habitability to climate, as shallow subsurface environments would be strongly affected by surface atmospheric conditions and deep subsurface environments may retain stable conditions regardless of surface climate.

(2) To what extent can the distribution of habitable environments be used to infer global trends? Understanding the global evolution of any aspect of the Mars system requires integrating preserved variations in local-to-regional environmental conditions across multiple, time-correlated sites. For example, does the inference of acidic diagenetic fluids at both the Opportunity and $\mathrm{Cu}-$ riosity sites $(62,104)$ reflect a global or local change in water composition $(6,14,105)$ ? Understanding regional variability is important for reconstructing paleoclimates, as global climatic changes should have regional effects depending on local topography, latitude, etc. (106).

(3) How representative are environments observed at landing sites? Landing sites are chosen based on exceptional features, which may not be representative of Mars as a whole. For example, 
hematite is abundant at Gale and Meridiani $(62,104)$, but orbital detections are rare on Mars (107). Thus, while these sites have been used as examples of Hesperian aqueous environments, it is uncertain if they are representative of broader Mars conditions. We need more information to extend results at these sites to a broader understanding of the evolution of habitable environments.

Diagenesis. (1) What is the distribution and extent of post-depositional alteration on Mars? Understanding diagenesis is critical for unraveling the history of environments at a site, but the timing and extent of post-depositional processes on Mars is poorly constrained. While diagenetic and low-grade metamorphic processes have been inferred based on the context of mineral detections from orbit (23-24), landed observations are needed to distinguish between depositional and post-depositional alteration minerals. For example, sulfates have been identified in sediments and fractures at Meridiani and Gale $(26,49)$ and hematite is present both as an early diagenetic cement and late diagenetic concretions at Gale $(62,108-112)$. This suggests that orbital sulfate and hematite detections could indicate either early surface or late subsurface fluids. Diagenesis is important for astrobiology, as it can enhance or diminish biosignature preservation, depending on the process and the biosignature (113). For example, MSL results demonstrated that sulfurization in the Pahrump Hills outcrop in Gale helped to preserve organics (114), but oxidizing and acidic diagenetic fluids inferred elsewhere in Mt. Sharp may have diminished organic preservation.

(2) Under what pressure/temperature (P/T) regime did post-depositional alteration occur? Mineral assemblages can be diagnostic of subsurface environmental conditions. The identification of prehnite provides evidence for high-T alteration $\left(200-400^{\circ} \mathrm{C} ; 115\right)$, while other assemblages indicate low-grade metamorphic or hydrothermal conditions during diagenesis $\left(\mathrm{T}<\sim 400^{\circ} \mathrm{C} ; \mathrm{P}<\sim 4\right.$ kbar; 24,85). Assemblages have been inferred from orbit under the assumption that minerals in close proximity are an assemblage (23-24), but, for robust constraints, mineral assemblages must be found in the same rock, so probing the nature of subsurface environments on Mars will require landed investigations. For example, identification of recrystallized hematite in local bleached zones by MSL has been used to infer warm fluids during late diagenesis (109-111).

Fluid properties. (1) What was the source of surface fluids? Surface fluids were likely produced through runoff from rain or snowmelt as well as groundwater upwelling $(13,10)$, but their relative contribution and the implications for climate are poorly constrained. Weathering minerals may be able to provide constraints on melt vs. rain (116), but additional work is needed to describe how other factors impact weathering products. Groundwater may have been a major water source (117), but analog studies have cast doubt on some geomorphic features attributed to groundwater (118). A better understanding of groundwater flow under realistic conditions on Mars is needed.

(2) What were the sources of subsurface fluids? Subsurface fluids may represent fluids released from burial and heating of wet sediments or hydrated minerals, or circulation of surface fluids. Understanding the sources of subsurface fluids is critical for understanding connectivity with surface environments, their relationship to atmosphere/climate, and nutrient sources. Redox-sensitive minerals can provide some constraints, as surface waters are more likely to be oxidizing (108); however, landed investigations are needed to confirm the diagenetic origin of these minerals.

(3) What was the chemistry of fluids? Aqueous geochemistry $(\mathrm{pH}$, redox, salinity, etc.) is a critical aspect of habitability that is linked with hydroclimate/atmospheric chemistry. However, key mineralogical indicators and their geologic context are not always discernible at current orbital scales, and geochemistry at outcrop scales is only possible with landed missions. Redox conditions are particularly important to constrain, as they enable key metabolic pathways and are an important control on biosignature preservation (113). Reducing fluids have been inferred on Mars (19,112), but it is unclear to what extent reducing environments require a biological contribution. 
Recommendations

Analog studies. Field and laboratory analog studies are critical for interpreting the geologic record of Mars and its habitability through time (113). Analog studies fill fundamental knowledge gaps and develop techniques critical to Mars exploration that are not common in traditional geology. We recommend that analog studies should be included within the Mars Exploration Program. Knowledge gaps that would benefit from analog studies include: the interface between surface and subsurface environments, the formation of sedimentary rocks on Mars, geological records of climate, and the origin of amorphous materials on Mars. More work is also needed to understand the limitation of using Earth-based frameworks for studying Mars, such as the effects of: long timescales for processes on a quiescent planet, redox processes in the absence of life, and atmospheric composition. Finally, finding good analogs is challenging, so we recommend that NASA support the development of a central repository for analog sites, data, and materials, such as the Terrestrial Analogs Data Portal in the Terrestrial Analogs Program at USGS.

Current missions. Current and upcoming missions will make important progress in the areas above, but only if those missions are adequately funded. We strongly recommend that the extended mission for MSL be funded as recommended in its external mission review. The extended mission is essential to explore the transition from wet to dry environments in Mt. Sharp - an exceptional environmental transformation preserved in an accessible sedimentary rock record. Recent budgets have threatened the continuance of MSL and at best would severely cripple operations, which will waste a valuable landed asset designed to interrogate past habitability. In addition, Mars 2020 will provide our first in situ look at Noachian habitable environments, and Mars Sample Return will answer critical questions about the geochemistry and timing of those environments.

Future missions. Community studies have identified the environmental record of Mars as a key target for future missions: NexSAG (119) and the mission concept MORIE (Calvin et al. white paper) both identified "environmental transitions" as a key orbital science goal, to be addressed using high resolution imaging with SWIR/TIR spectroscopy for mineral mapping. MASWG (Jakosky et al. white paper) specifically highlighted that "Mars has a uniquely accessible archive of the long-term evolution of a habitable planet" as the key motivation for a rejuvenated Mars Exploration Program. MASWG suggested a possible mission arc to study habitable environments, including an orbiter to acquire high spatial resolution mineralogy, a mobile lander(s) for surface exploration of multiple promising outcrops, and eventually a larger mission to conduct detailed habitability and astrobiology studies. We agree with this approach, and we recommend that the most critical mission components to achieve in the next decade are orbital high-resolution spectral imaging and landed mobile missions to multiple ancient environments.

Coordinated orbital SWIR (hyperspectral, 1.8-2.6 $\mu \mathrm{m},<10 \mathrm{~m} / \mathrm{pixel}$ ) and TIR (tens of bands, 8$25 \mu \mathrm{m},<100 \mathrm{~m} /$ pixel) imaging would allow discernment of key geochemical indicator minerals as well as their abundance. Multispectral imagers (tens of bands, 0.3-1.5 $\mu \mathrm{m},<1 \mathrm{~m} /$ pixel) would enable detection of many redox minerals, local heterogeneity, and link outcrops to detailed SWIR/TIR mineralogy. Characterizing global trends in aqueous processes on Mars requires an improved understanding of mineral assemblages and their abundances. High resolution color and spectral data is essential for construction of detailed stratigraphies from orbit in order to identify locations with the most promising records of ancient environments and environmental transitions.

As the Mars Exploration Program has demonstrated, the detail provided by landed missions will be necessary to accurately constrain the geologic history of a site. We strongly advocate for the concept of small vehicles with lateral mobility sent to multiple landing sites across the planet in order to most efficiently piece together the details of the evolution of habitable environments 
on Mars. These missions could be in the form of either rovers or aerial vehicles (Bapst/Rapin white papers), equipped with a payload capable of, at a minimum, imaging, spectral mapping (hyper- or multispectral) and geochemistry (e.g., APXS, SuperCam). Bulk mineralogy would be ideal if the technology can be miniaturized (e.g., arm-mounted CheMin). In addition, quantitative geochronology of sites preserving key environmental transitions will be critical to tie these events in time (Cohen et al. white paper). Additional orbital data will be necessary for identifying specific locations that preserve key environmental transitions and that allow surface access to records of subsurface environments. As discussed by MASWG, this multi-site approach will require significant technology development, and we strongly recommend that a robust Mars Exploration Program should enable progress in small mobile platforms and cheaper ways to access the surface.

References: (1) MEPAG (2020) Goals. (2) Ehlmann et al. (2016) 10.1002/2016JE005134. (3) Bibring et al. (2006) 10.1126/science 1122659. (4) Murchie et al. (2009) 10.1029/2009JE003342. (5) Grotzinger \& Milliken (2012) SEPM SP 102. (6) Irwin et al. (2005) 10.1029/2005JE002460. (7) Fassett \& Head (2008) 10.1016/j.icarus.2007.12.009. (8) Burr et al. (2009) 10.1016/j.icarus.2008.10.0 14. (9) Metz et al. (2009) 10.1029/2009JE003365. (10) Ansan \& Mangold (2013) 10.1002/igre.20117. (11) Matsubara et al. (2013) 10.1002/jgre.20081. (12) Irwin et al. (2015) 10.1016/j.geomorph.2014.10.012. (13) Wray et al. (2011) 10.1029/2010JE003694. (14) Horvath \& Andrews-Hanna (2017) 10.1002/2017GL74654. (15) Michalski et al. (2013) 10.1038/NGEO1706. (16) Carter et al. (2015) 10.1016/j.icarus.2014.11.011. (17) Gaudin et al. (2011) $10.1016 /$ j.icarus.2011.09.004. (18) Farrand et al. (2014) $10.1016 /$ j.icarus.2014.07.003. (19) Bishop et al. (2013) 10.1016/j.pss.2013.05.006. (20) Bishop \& Rampe (2016) 10.1016/j.epsl.2016.04.0 31. (21) Bishop et al. (2018) 10.1038/s41550-017-0377-9. (22) Arvidson et al. (2014) 10.1126/science.1248097. (23) McSween et al. (2015) 10.1111/maps.12330. (24) Ehlmann et al. (2011) 10.1346/CCMN.2011.0590402. (25) Michalski et al. (2017) 10.1038/nc omms15978. (26) Kronyak et al. (2019) 10.1029/2018EA000482 (27) Sun et al. (2019) 10.1016/j.icarus.2018.12.030 (28) Loizeau et al. (2015) 10.1002/2015JE004894. (29) Saper \& Mustard (2013) 10.1002/grl.50106. (30) Michalski et al. (2013) 10.1038/ngeo17 06. (31) Tarnas et al. (2018) 10.1016/j.epsl.2018.09.001. (32) Onstott et al. (2019) $10.1089 /$ ast.2018.1960. (33) Milliken et al. (2014) 10.1002/2013GL059097. (34) Day et al. (2019) 10.1029/2019JE006226. (35) Davis et al. (2016) 10.1130/G38247.1. (36) Cardenas et al. (2018) 10.1130/B31567.1. (37) DiBiase et al. (2013) 10.1002/jgre.20100. (38) Goudge et al. (2017) 10.1016/j.epsl.2 016.10.056. (39) Kite et al. (2013) 10.1016/j.icarus.2013.03.029. (40) Kite et al. (2017) 10.1002/2017GL0 72660 (41) Kite et al. (2019) 10.1126/sciadv.aav7710. (42) Kite et al. (2014) 10.1038/ngeo2137. (43) Goudge et al. (2018) 10.1016/j.icarus.2017.09.034. (44) Grotzinger et al. (2014) 10.1126/science.1242777. (45) Grotzinger et al. (2015) 10.1 126/science.aac7575. (46) Vaniman et al. (2014) $10.1126 /$ science.1243480. (47) McLennan et al. (2014) 10.1126/science.1 244734. (48) Bristow et al. (2018) $10.1126 /$ sca dv.aar3330. (49) Rapin et al. (2019) 10.1038/s41561-019-0458-8. (50) Mangold et al. (2018) 10.1016/j.icarus.2018.11.004. (51) Mangold et al. (2019) 10.1016/j.icarus.2018.11.004. (52) Rivera-Hernández et al. (2020) 10.1029/2019JE006230. (53) Milliken et al. (2010) 10.1029/2009GL041870. (54) Horgan \& Rice (2015) LPSC46, \#2943. (55) Sheppard et al. (2020) 10.1002/essoar.105023 76.1. (56) Palucis et al. (2016) 10.1002/2015JE004905. (57) Martin et al. (2017) 10.1002/2017JE005445. (58) Ehlmann \& Edwards (2014) 10.1146/annurev-earth-. (59) Viviano-Beck et al. (2014) 10.1002/2014JE004627. (60) Farrand et al. (2009) 10.1016/j.icarus 2009.07.014. (61) Carter et al. (2015) 10.1016/j.icarus.2015.01.020. (62) Rampe et al. (2017) 10.1016/i.epsl.2017.04.021. (63) Franz et al. (2014) 10.1038/nature13175. (64) Wong et al. (2020) 10.1029/2019JE006304. (65) Sutter et al. (2017) $10.1002 / 2016 \mathrm{JE}$ 005225. (66) Franz et al. 2020 10.1038/s41550-019-0990-x. (67) Amador et al. (2018) 10.1016/j.icarus.2018.03.021. (68) Jakosky (2019) $10.1016 /$ j.Pss.2019.06.002. (69) Mahaffy et al. (2015) $10.1126 /$ science.1260291. (70) Potter-McIntyre (2014) $10.1089 /$ ast.2 013.0974. (71) Clark et al. (2007) 10.1029/2006JE002756. (72) Morris et al. (2010) 10.1126/science.1189667. (73) Ruff \& Farmer (2016) 10.1038/ncomms13554. (74) Grotzinger \& Milliken (2012) 10.2110/pec.12.102.0. (75) Cannon et al. (2017) 10.1038/nature

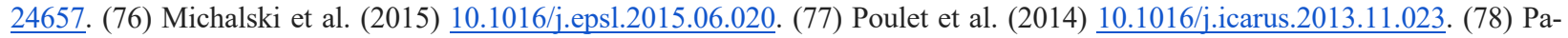
lumbo \& Head (2018) 10.1111/maps.13001. (79) Peretyazhko et al. (2015) 10.1016/j.gca.2015.10.012. (80) Ehlmann et al. (2008) 10.1126/science.1164759. (81) Archer et al. (2020) LPSC51 \#2709. (82) Horgan et al. (2020) 10.1016/j.icarus.2019.113526. (83) Kelemen et al. 2020 LPSC51 \#1213. (84) Brown et al. (2010) 10.1016/j.epsl.2010.06.018. (85) Viviano et al. (2013) 10.21002/jgre. 20141. (86) Kelemen et al. 2020 LPSC51 \#1213. (87) Ramirez \& Craddock (2018) 10.1038/s41561-018-0093-9. (88) Ramirez et al. (2020) 10.1029/2019JE006160. (89) Hynek et al. (2010) 10.1029/2009JE003548. (90) Wordsworth et al. (2013) $\underline{10.1016 / j . i c a r u}$

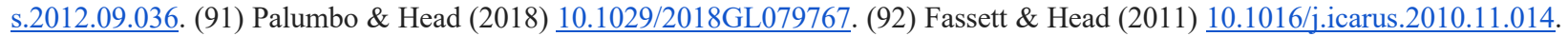
(93) Cassanelli \& Head (2015) 10.1016/j.icarus.2015.03.004. (94) Wordsworth (2016) 10.1146/annurev-earth-060115-012355. (95) Grant et al. (2014) 10.1002/2013GL058909. (96) Ehlmann \& Buz (2015) 10.1002/2014GL062553. (97) Palucis et al. (2020) $\underline{10.1016 / j . i c a r u s .2020 .113623}$. (98) Sun \& Milliken (2018) 10.1029/2018GL078494. (99) Stack et al. (2019) 10.1111/sed.12558. (100) Edgar et al. (2020) 10.1029/2019JE006307. (101) Jerolmack et al. (2004) 10.1029/2004GL021326. (102) Lapotre \& Ielpi (2020) 10.1029/2019AV000141. (103) Tornabene et al. (2013) 10.1002/igre.20082. (104) McLennan et al. (2005) 10.1016/i.epsl.20 05.09.041. (105) Andrews-Hanna et al. (2007) 10.1038/nature05594. (106) Bouley et al. (2016) 10.1038/nature17171. (107) Thomas et al. (2019) LPSC50 \#2065. (108) Hurowitz et al. (2017) 10.1126/science.aah6849 (109) Fraeman et al. (2020) LPSC51 \#1677. (110) Horgan et al. (2020) 10.1002/essoar.10501380.1. (111) Rampe et al. (2020) 10.1029/2019JE006306. (112) L'Haridon et al. (2020) LPSC50 \#6079. (113) Hays et al. (2016) 10.1089/ast.2016.1627. (114) Eigenbrode et al. (2018) 10.1126/science.aas91 85. (115) Ehlmann et al. (2009) 10.1029/2009JE003339. (116) Rutledge et al. (2018) 10.1029/2018GL078105. (117) AndrewsHanna \& Lewis (2012) 10.1029/2010JE0. (118) Irwin et al. (2014) 10.1016/j.icarus.2 014.08.012. (119) MEPAG (2015) NEXSAG. 